\title{
Preoperative Evaluation and Investigations for Maxillofacial Surgery
}

\author{
B. Krishnan and Satyen Parida
}

\subsection{Introduction}

A meticulous preoperative evaluation with the intention of identifying modifiable clinical conditions and understanding the risk stratification is imperative for all patients being subjected to any invasive procedure, inclusive of oral and maxillofacial surgery. Preoperative evaluation of such patients would of course differ, often dictated by age and the overall health condition that they present with. The choice of anesthesia may vary depending on the surgical procedure intended: general anesthesia, conscious sedation, regional blocks, local anesthesia, or various combinations of all of these. The current chapter focuses on the preoperative evaluation of patients posted for maxillofacial surgery under a general anesthetic in a formal operating room setting. Nevertheless, it is essential to realize that a preoperative evaluation is more or less standardized, irrespective of the type of surgery and practice (office-based dentoalveolar surgery, trauma, orthognathic surgery, elective esthetic surgery, craniofacial surgery, etc.) and anesthesia being planned. The goals of preoperative evaluation are summarized in Fig. 2.1 [1]. The intention is to minimize perioperative morbidity and mortality, to the best possible extent. The major step toward this goal requires the surgeon to be conscientiously aware of the general condition and clinical state of the patient so that in the eventuality of a critical event, he can intervene effectively and pertinently. This would involve modifying and customization of the patient's overall management to ensure that perioperative adverse events are avoided.

B. Krishnan $(\varangle)$

Department of Dentistry, Jawaharlal Institute of Postgraduate Medical Education and Research (JIPMER), Puducherry, India

S. Parida

Department of Anesthesiology and Critical Care,

Jawaharlal Institute of Postgraduate Medical Education and

Research (JIPMER), Puducherry, India

\subsection{Preoperative Evaluation}

The process of preoperative evaluation often begins with a standard history-taking and physical examination for the particular patient. A detailed history of prior medical problems, any previous surgical procedures, family, personal, and social history, any chronic medications and allergies or addictions needs to be obtained. In addition, it is worthwhile obtaining details about the patient's family physician if any, whether he has health insurance, the employer's details if the patient is employed, contact details of relatives, and any other information that may be deemed appropriate. The maxillofacial surgeon has to judge how the medical problems of the patient will affect perioperative care, and conversely, how perioperative events would influence the management of the patient's medical conditions. The patient's comorbidities could result in a significant physiologic decompensation due to myriad factors such as stress of the surgical procedure, original goals of the surgical intervention not being adequately met, and potential interactions between the regular medications that a patient may be on or with drugs that may be required to be instituted as part of the perioperative protocol. The maxillofacial surgeon should, therefore, be cognizant of what could possibly go wrong, and how those situations could be remedied. Also, it is critical that the surgeon be aware of all the medications (antidiabetic drugs, anticoagulants, antiplatelets, etc.) and medical comorbidities (cardiopulmonary, central nervous system, hepatic and renal disease, etc.) that the patient may be exposed to that could threaten the safe performance of the surgical procedure and have an adverse impact during the postoperative period. It is essential that every member of the perioperative team must be able to perform an independent assessment of the patient. This does not imply being ignorant of the other person's assessment since information needs to be shared by all components of the team, but each member should have his own way of assimilating such information by adding to it by his own independent assessment. This is important because 


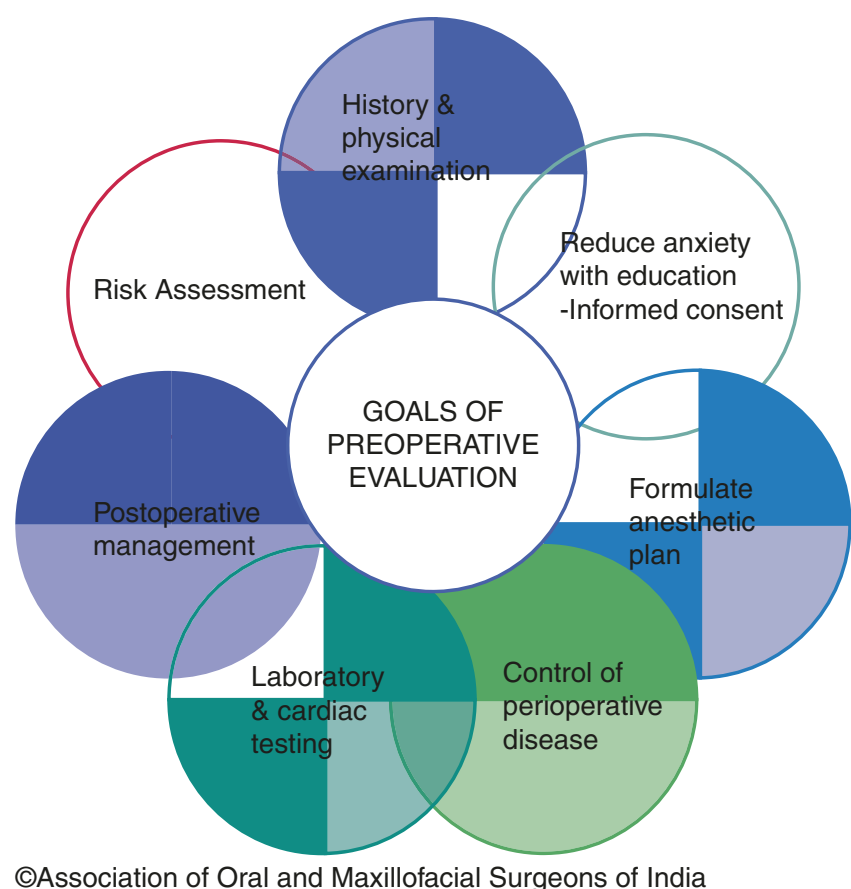

CAssociation of Oral and Maxillofacial Surgeons of India

Fig. 2.1 Goals of preoperative evaluation

every specialty would have its independent perspective, about the patient, although they would all be in the latter's best interests. For example, the surgeon might look upon older age in terms of postoperative functional recovery, while the anesthesiologist would be contemplating the various comorbidities that could be associated. Accordingly, each specialist would have his own concerns regarding the state of the patient, and therefore, the preoperative assessment provides a meeting ground for all these concerns after everyone has performed his own independent assessment. The use of a medical history questionnaire provides the maxillofacial surgeon with valuable information about the physical and psychological condition of the patient. Any medical history questionnaire can either be extremely useful or totally worthless and its ultimate value depends upon the ability of the surgeon to interpret the significance of the answers and to elicit additional information through physical examination and dialogue history. A prototype of a health history questionnaire is seen in Table 2.1 [2].

A preoperative physical examination begins with a recording of the patient's vital signs. The oral and maxillofacial system, by virtue, of the proposed surgical site, receives close attention by the surgeon. Of particular interest for the surgeon would be recognizing potential difficulties in surgical access and the need for alternative strategies and additional surgeries (e.g., bone grafting/skin grating). The cardiopulmonary examination assesses the rate and rhythm, murmurs, wheezing, rhonchi, and stridor. The musculoskeletal examination will include a range of neck motion and
Table 2.1 Preoperative medical evaluation questions for a healthy patient

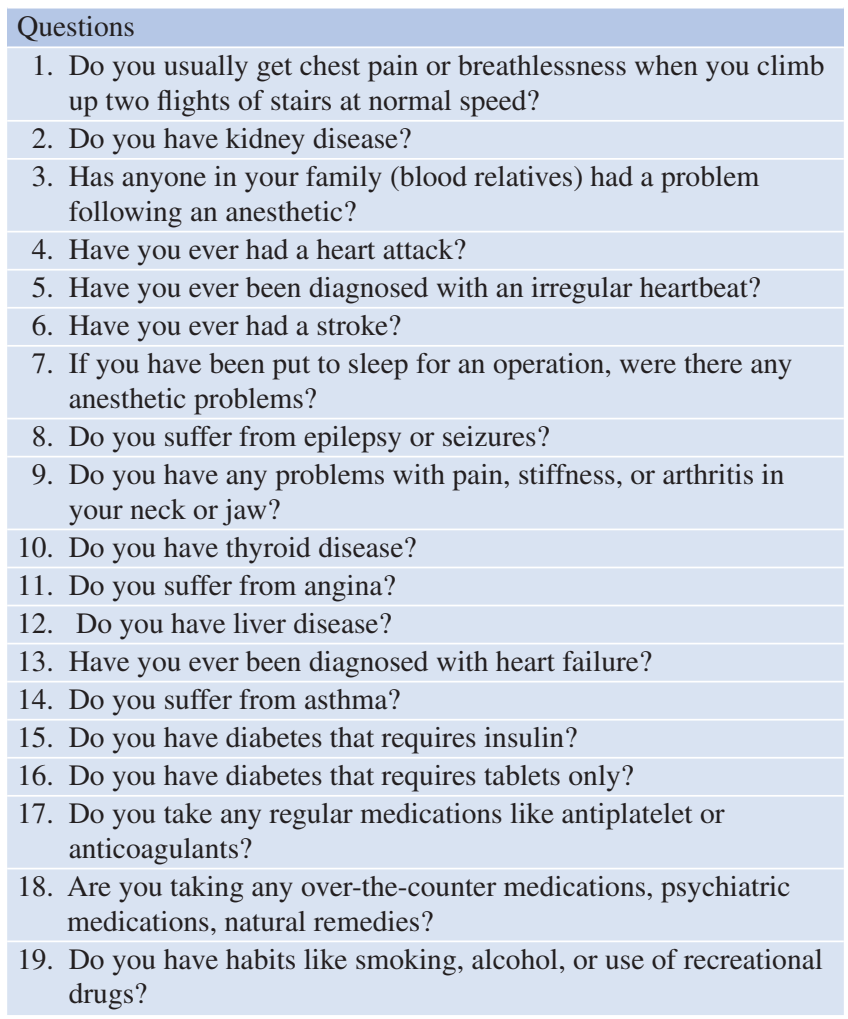

potential donor site evaluation. Finally, a neurological examination would assess the patient's mental status and any signs of pre-existing nerve or head injuries. A detailed description of the examination of all systems is beyond the scope of this chapter and the reader is advised to consult the references at the end of this chapter [1,3-5].

Once the history and physical examination are complete, the clinician would be in a situation to risk stratifying the patient according to the American Society of Anesthesiologists' (ASA) classification system (please see chapter on Ambulatory anesthesia for ASA table). Further investigations or specialist consultation is determined by the ASA status. This is especially imperative in patients with ASA Class III or IV who need to be further evaluated for their ability to withstand the proposed surgical procedure. Such patients, almost always, require inpatient admission due to a higher risk of perioperative adverse events. Patients of ASA Class V are extremely moribund and would therefore invariably require admission irrespective of the surgical procedure involved. It is extremely uncommon for a patient in this category to undergo any maxillofacial surgical procedure.

Preoperative risk stratification can also include a surgical classification system [6] (Table 2.2). This classification is quite useful for healthy patients undergoing a surgical procedure, as risk assessment in these patients is now determined solely by the nature of the procedure rather than the ASA 
Table 2.2 Cardiac Risk Stratification for Noncardiac Surgical procedures

\begin{tabular}{l|l}
$\begin{array}{l}\text { Cardiac risk } \\
\text { stratification }\end{array}$ & Procedure examples \\
\hline $\begin{array}{l}\text { High Risk } \\
(>5 \%)\end{array}$ & $\begin{array}{l}\text { Aortic, Major vascular Surgery, Peripheral } \\
\text { vascular Surgery }\end{array}$ \\
$\begin{array}{l}\text { Intermediate } \\
\text { Risk }(1-5 \%)\end{array}$ & $\begin{array}{l}\text { Intraperitoneal and Intrathoracic Surgery, Carotid } \\
\text { endarterectomy, Head and neck surgery, } \\
\text { Orthopedic Surgery, Prostate Surgery. }\end{array}$ \\
\hline Low Risk $(<1 \%)$ & $\begin{array}{l}\text { Endoscopic procedures, Superficial procedures, } \\
\text { Cataract surgery, Breast surgery, Ambulatory } \\
\text { surgery }\end{array}$ \\
\hline
\end{tabular}

classification [7]. A similar surgical severity classification for oral and maxillofacial procedures has also been offered [8].

The preoperative assessment for oral and maxillofacial surgery will also require appropriate radiologic imaging for diagnostic information and treatment planning. These include panoramic, periapical, and occlusal radiographs, ultrasound, CT and MRI imaging. The clinical evaluation and imaging studies should help in the formation of a surgical plan, as to whether the surgery is going to be a simple or a complex one, what kind of instrumentation will be required for the same, or whether it would require the expertise of other surgical specialties such as Ophthalmology, ENT, and Plastic Surgery. The anesthesia team can also examine these imaging modalities to identify potential difficulties that may be encountered while intubating and securing the airway.

\subsection{Laboratory Investigations}

Preoperative patient assessment, ASA physical status, and severity of the proposed oral and maxillofacial surgical procedure guide the clinician to select the appropriate adjunctive studies for treatment planning. The ordering of adjunctive laboratory and radiological investigations is often determined by a complex interplay of pre-existing attitudes, practice patterns learnt during training, ease of ordering, fear of medicolegal consequences, and patient requests. When ordering a test, clinicians are attempting to discriminate between patients who have normal values and those who have abnormal ones. This is used to determine the possibility of the existence of a pathological condition that could impact the proposed surgical and anesthetic plan. The "normal" reference range for many clinical lab tests excludes the upper and lower $2.5 \%$ of results, and therefore $5 \%$ of normal individuals will obtain an "abnormal" result. While these "abnormal results" can be ignored, sometimes they are not and the result is an additional unnecessary investigation. Clinicians must be confident that there is a clinical justification for the actual need for the test. Studies indicate that less than $0.25 \%$ of all "abnormal" results of preoperative tests before an elective surgery influences the perioperative management [9]. "Routine" investigations, viz., those carried out preoperatively on all patients, and not directly related to the planned surgical procedure or the patient's physical condition, are not recommended $[10,11]$. Age-based criteria are controversial as test abnormalities are common in older patients but are not as predictive of complications as information gained from a detailed history and physical examination. "Cost consciousness" and "stewardship of resources" also have to be considered by the clinician as the commonly ordered "battery of tests," though relatively inexpensive by themselves, can contribute to a significant proportion of healthcare expenditure as a result of the frequency with which they are ordered [12]. Beyond economics, the costs borne by patients include discomfort of needle sticks and blood loss with repeated phlebotomy, exposure to radiation, and additional unnecessary workup of spurious results. Good communication between the surgeon, medical consultant, anesthesia team, and the patient is essential when considering preoperative testing that may affect the timing of the surgery. Fit, young patients who are scheduled for outpatient surgery or low-risk surgery generally do not require any routine preoperative testing and, in the pediatric age group, a thorough clinical examination has been found to be of greater value $[13,14]$. Routine urine or blood testing of the pediatric patient is not clinically warranted without a specific indication [15]. In general, test results within the past 6 months are acceptable if the patient's history has not changed. If the patient's condition has changed in the interim, adjunctive tests within the past 2 weeks are more favored [1].

The American Association of Oral and Maxillofacial Surgeons (AAOMS) recommendations on adjunctive studies prior to oral and maxillofacial surgery include, but are not limited to, both laboratory and imaging investigations [16] (Table 2.3). Similarly, algorithms based on the ASA classification, surgical procedure, and age of the patient are also available to assist the clinician to choose the appropriate adjunctive preoperative tests $[17,18]$.

While most standard texts would lead one to believe that extensive investigations in patients who are supposedly normal on history and clinical appraisal are not instrumental in improving outcomes or economically justified, it must be remembered that these perceptions are built upon data accumulated from the West, where regular health checkups and assessments are the norms. In contrast, in countries like India, a patient's first visit to a hospital may be for the surgery he is being posted for. Patients may also not be aware of existing comorbidities or details of any earlier interventions. This often leaves the clinician with no other option than to do order a "battery" of the so-called routine investigations to screen out potential pathological conditions. Ultimately, the choice of what laboratory investigations a patient may be subjected to is decided by the particular operating unit. It is important that such practices be customized to the population that the hospital caters to, and must be based on reasonable risk-benefit and cost analysis. 
Table 2.3 Indications for commonly ordered preoperative laboratory tests based on specific findings during history and physical examination (independent of patient age, American Society of Anesthesiologists' classification, or surgical procedure)

\begin{tabular}{|c|c|}
\hline Investigations & Indications \\
\hline \multicolumn{2}{|l|}{ Laboratory investigations } \\
\hline $\begin{array}{l}\text { 1. Complete Blood Counts: } \\
\text { WBC count }\end{array}$ & $\begin{array}{l}\text { Patients with infection/fever } \\
\text { On chemotherapy } \\
\text { Myeloproliferative disorders } \\
\text { Immune-compromised states (HIV) } \\
\text { History of cancer, chemotherapy, or radiation treatment }\end{array}$ \\
\hline Platelet count & $\begin{array}{l}\text { History of low platelets } \\
\text { Bleeding history } \\
\text { Thrombopathy } \\
\text { Pregnancy } \\
\text { Autoimmune disease } \\
\text { Splenectomy } \\
\text { Liver disease }\end{array}$ \\
\hline Hemoglobin/Hematocrit & $\begin{array}{l}\text { Anticipated blood loss greater than } 500 \mathrm{ml} \\
\text { Suspicion of anemia } \\
\text { Recent chemotherapy/Radiotherapy (within } 2 \text { months) } \\
\text { Renal disease } \\
\text { Active cardiac symptoms } \\
\text { Recent blood loss } \\
\text { Sickle cell anemia/thalassemia } \\
\text { Recent autologous blood transfusion } \\
\text { Older patients undergoing major surgery }\end{array}$ \\
\hline $\begin{array}{l}\text { 2. Chemistry: } \\
\text { Electrolytes/BUN/Creatinine }\end{array}$ & $\begin{array}{l}\text { Chronic Kidney Disease } \\
\text { Liver cirrhosis } \\
\text { Medications (diuretics, ACE inhibitor, digoxin) } \\
\text { Diabetes mellitus } \\
\text { Malnutrition } \\
\text { Dehydration } \\
\text { Infections } \\
\text { On renal dialysis- }-\mathrm{K}^{+} \text {tested immediately prior to surgery }\end{array}$ \\
\hline Creatinine & $\begin{array}{l}\text { Older patients undergoing intermediate-or-high-risk surgery } \\
\text { Patient for whom nephrotoxins will be used } \\
\text { When large fluid shifts or hypotension is likely }\end{array}$ \\
\hline $\begin{array}{l}\text { Glucose-(Fasting/Random/ } \\
\text { Postprandial/Glucose Tolerance } \\
\text { Test (GTT) /HbA1C) }\end{array}$ & $\begin{array}{l}\text { Diabetes mellitus } \\
\text { Long-term steroid medications } \\
\text { Liver cirrhosis } \\
\text { Pregnancy } \\
\text { Infections } \\
\text { Pancreatic disease } \\
\text { Adrenal disease } \\
\text { Pituitary disease } \\
>45 \text { Years of age with no prior history of diabetes mellitus }\end{array}$ \\
\hline $\begin{array}{l}\text { 3. Coagulation profile } \\
\text { Prothrombin Time (PT) } \\
\text { International Normalized Ration } \\
\text { (INR) } \\
\text { Partial Thromboplastin Time (PTT) }\end{array}$ & $\begin{array}{l}\text { Personal/ family history of bleeding diathesis or clotting disorders } \\
\text { Evidence of purpura or petechiae } \\
\text { On current or recent anticoagulation medications (warfarin/heparin) } \\
\text { Liver disease } \\
\text { Abnormal liver function (jaundice) } \\
\text { Alcoholism } \\
\text { Severe malnutrition } \\
\text { Chronic renal failure } \\
\text { History of stroke } \\
\text { Autoimmune disorders }\end{array}$ \\
\hline $\begin{array}{l}\text { PT (for warfarin) } \\
\text { PTT (for heparin) }\end{array}$ & $\begin{array}{l}\text { Repeated on the evening before/morning of the surgery to document normal coagulation parameters after } \\
\text { stopping these medications, if indicated. }\end{array}$ \\
\hline Bleeding Time (BT) & Not a reliable preoperative indicator of platelet function. Not recommended for presurgical workup \\
\hline Clotting Time (CT) & $\begin{array}{l}\text { Time consuming, poor reproducibility, insensitive to high doses of heparin, and sensitive in only extreme } \\
\text { factor deficiencies } \\
\text { Not of much value in modern laboratory settings }\end{array}$ \\
\hline
\end{tabular}


Table 2.3 (continued)

Investigations

4. Liver function tests

5. Type, screen, and cross-sensitivity

6. Pregnancy testing (Serum/Urine)

7. Arterial blood gases (ABG)

8. Pulmonary function tests

9. Urinalysis

10. Blood cultures

Imaging

1. Chest X Ray

\section{12 Lead ECG}

3. Echocardiogram
Indications

\section{Cirrhosis}

Hepatitis

Jaundice

Hepatomegaly

Alcohol abuse

Pancreatic disease

History of cancer

Easy bleeding and bruising

Malnutrition

Patients with bleeding or coagulation disorders

Surgeries with a potential for blood loss $(>500 \mathrm{ml})$ even if transfusion is not expected.

This may help minimize the risk of later transfusion reaction.

Premenopausal women of child-bearing age who have not had tubal ligation or hysterectomy (Urine

Human Chorionic Gonadotropin-HCG).

History and physical examination are insufficient to determine early pregnancy, and patients are often unreliable in suspecting that they may be pregnant. Management protocols often change if it is discovered that the patient is pregnant.

Only if suspicion for hypoxemia or $\mathrm{CO}_{2}$ retention that would affect postoperative management.

Previously unknown obstructive lung disease

Severe asthma

Symptomatic COPD

Scoliosis

Active symptoms of Urinary tract infection (UTI), dysuria

No good evidence that preoperative abnormal urinalysis is associated with any postoperative complication in nonurinary tract surgery.

Only if clinical suspicion of septicemia.

Significant risk factors for postoperative pulmonary complications (severe/uncontrolled COPD, active pulmonary disease/symptoms, abnormal lung sounds on physical examination, recent pneumonia) with no previous chest X-ray for past 1 year may warrant preoperative chest X-ray irrespective of age.

Asymptomatic patients older than 50 years with no risk factors-insufficient evidence for ordering a chest X-ray.

Should not be considered as an unequivocal indication for extremes of age, smoking, COPD, stable cardiac disease, and recent resolved upper Respiratory Tract Infection (RTI).

Without symptoms or pertinent medical history, abnormal chest X rays do not predict a worse clinical outcome.

History of cardiac disease (coronary artery disease, significant arrhythmia, structural heart disease, compensated or prior heart failure)

Peripheral artery disease

Chest pains

Lung disease

Morbid obesity

History of stroke/cerebrovascular disease

Diabetes mellitus

Renal insufficiency

Patient is having an intermediate- or high-risk surgery

Consider in high-risk group based on epidemiology (males $>40$ years and females $>50$ years of age)

Recent myocardial infarction

Congestive heart failure

Abnormal ECG

Unstable angina

Significant arrhythmias

Severe valvular heart disease

Previous history of coronary artery disease

Dyspnea of unknown origin

History of heart failure with progressive symptoms

Unstable cardiomyopathy 


\subsection{Preanesthetic Evaluation}

The preanesthetic evaluation aims at getting a favorable outcome, from the points of view of both the surgeon and the anesthesiologist by focusing on risk stratification and modification, and by developing a plan that appropriately addresses the risk-benefit ratio from the procedure, and also explores the other options available. It involves putting together all the elements of the patient's history, physical examination, medications, allergies, laboratory studies, and certain examinations of interest to the anesthesiologist (e.g., airway). This information is assimilated, and decisions are made regarding the plan for anesthetizing the patient once the full picture emerges. This, however, is a dynamic process, and hence accommodates changes in the plan, if new information surfaces, in the best interests of the patient and surgeon. It is also important to be aware of the strengths and limitations of the set-up in which the particular surgical intervention is being undertaken. Further, the preanesthetic evaluation also attempts to allay patient anxiety regarding the procedure, takes necessary steps for risk reduction and to get informed consent. Finally, it leads to the choice of the anesthetic plan to be followed, guided by the risk factors uncovered by the medical history, which covers the entire perioperative period, inclusive of a plan as to where the patient will be cared for postoperatively, and what kind of pain relief he/she should receive after surgery.

\subsection{Aspects of Evaluation Unique to Anesthesia}

The perspective of each specialty also drives the way physical examination is conducted by each of them. All clinicians learn early as part of their training that relevant positive and negative points not only direct the elicitation of history but also examination. Airway examination is something that is uniquely specific to anesthesiologists with the intention of predicting a difficult airway, that is, one that might result in difficulty in ventilating or intubating the patient, or both. The ASA task force on the management of the difficult airway stresses importance on the physical examination of the airway [19]. While no single method can be deemed to be foolproof in this regard, the anesthesiologist is trained to examine different aspects as well as scoring systems to be able to make the assessment of a difficult airway [20-22]. The mne- monic LEMON is a helpful tool for assessing the potential for difficult intubation [23, 24] (Fig. 2.2). The Mallampati classification, which relates tongue size to pharyngeal size, is a common component of a thorough airway examination [25]. The examination is performed with the patient in the sitting position, the head held in a neutral position, the mouth wide open, and the tongue protruding to the maximum. The subsequent classification is assigned according to the pharyngeal structures that are visible: (Fig. 2.3).

Class I: visualization of the soft palate, fauces, uvula, and anterior and posterior pillars.

Class II: visualization of the soft palate, fauces, and uvula.

Class III: visualization of the soft palate and the base of the uvula.

Class IV: soft palate not visible at all.

The important aspects of the airway, which can affect intubation, include the degree of mouth opening, the size of the tongue in relation to other oral structures, and the capability to align the oral, pharyngeal, and laryngeal axes in more or less one straight line, with the patient's head and neck in the sniffing position. While the Mallampati classification relates to soft-tissue sizes and their relationships with each other, apart from mouth opening, there are several other anatomical features that need to be considered in the evaluation of the airway (Table 2.4). Examination of systems, other than the cardiopulmonary system, is dependent upon the proposed surgical procedure and planned anesthesia, along with the clinical features of the patient. For example, a patient with a Le Fort II or III fracture may require a careful neurological and ophthalmological examination. Documentation of the findings of history and physical examination must focus on the positive and negative findings after integrating information from the surgeon, patient, and other sources.

\subsection{Assessment of the Pediatric Patient}

The objective of preoperative assessment in the apparently healthy child is to discover medical or anatomic issues, hitherto not acknowledged, that will escalate the risk of surgery and anesthesia. Special attention is warranted by virtue of specific anatomical and physiological differences from the adult patient. Additional risk factors in pediatric patients, apart from the surgery-specific and patient-specific risks, 
Fig. 2.2 LEMON

Assessment for difficult intubation

\begin{tabular}{|c|c|c|}
\hline Physical Signs & Less difficult airway & More difficult airway \\
\hline Look externally & $\begin{array}{l}\text { Normalface and neck } \\
\text { No face or neck pathology }\end{array}$ & $\begin{array}{l}\text { Abnormally face shape } \\
\text { Sunken cheeks } \\
\text { Edentulous } \\
\text { Buck teeth } \\
\text { Receding mandible } \\
\text { Narrow mouth } \\
\text { Obesity } \\
\text { Face or neck pathology }\end{array}$ \\
\hline Evaluate $3-3-2$ rule & $\begin{array}{l}\text { Mouth opening }>3 \text { fingers } \\
\text { Hyoid-chin distance }>3 \text { fingers } \\
\text { Thyroid cartilage to mouth floor } \\
\text { distance }>2 \text { fingers }\end{array}$ & $\begin{array}{l}\text { Mouth opening }<3 \text { fingers } \\
\text { Hyoid-chin distance }<3 \text { fingers } \\
\text { Thyroid cartilage to mouth } \\
\text { floor distance }<2 \text { fingers }\end{array}$ \\
\hline Mallampatti scale & $\begin{array}{l}\text { Class I and II (Can see the soft palate, } \\
\text { uvula, fauces, } \pm \text { facial pillars) }\end{array}$ & $\begin{array}{l}\text { Class III and IV (Can only see } \\
\text { the hard palate } \pm \text { soft palate } \pm \\
\text { base of uvula) }\end{array}$ \\
\hline Obstruction & None & $\begin{array}{l}\text { Pathology within or } \\
\text { surrounding the upper airway } \\
\text { (e.g., peritonsillar adscess, } \\
\text { epiglottis, retropharyngeal } \\
\text { abscess) }\end{array}$ \\
\hline Neck mobility & $\begin{array}{l}\text { Can flex and extend the neck } \\
\text { normally }\end{array}$ & $\begin{array}{l}\text { Limited range of motion of the } \\
\text { neck }\end{array}$ \\
\hline
\end{tabular}

CAssociation of Oral and Maxillofacial Surgeons of India

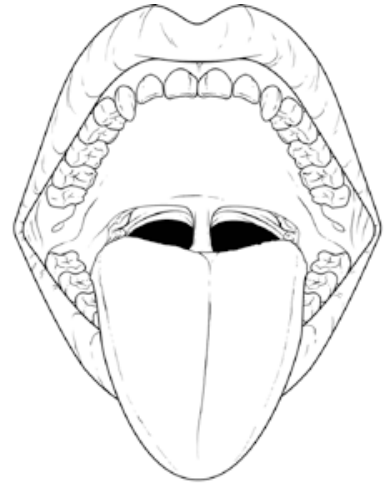

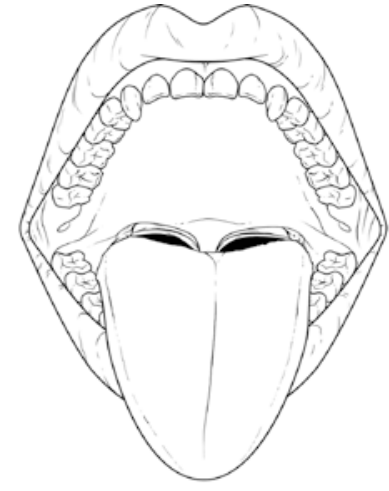

III

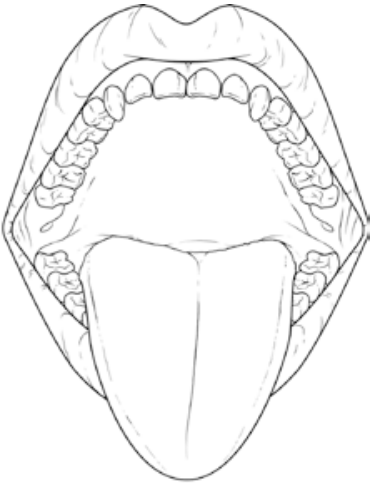

IV

Fig. 2.3 Mallampati Classification for assessment for intubation. Class I: Cmplete visualization of the soft palate. No additional difficulty. Class II: Complete visualizatin of the uvula. No additional difficulty. Class III: Visualization f only the base of the uvula. Moderate difficulty. Class IV: Soft palate is not visible at all. Severe difficulty 
Table 2.4 Airway compromising conditions

- Congenital
- Pierre-Robin syndrome: micrognathia, macroglossia, cleft soft
palate
- Treacher-Collins syndrome: auricular and ocular defects, malar
and mandibular hypoplasia
- Goldenhar's syndrome: auricular and ocular defects, malar and
mandibular hypolasia
- Down's syndrome: poorly developed or absent bridge of the
nose, macroglossia
- Kippel-Feil syndrome: congenital fusion of a variable number
of cervical vertebrae, restriction of neck movement
- Goiter: compression of trachea, deviation of larynx/trachea
- Infections
- Supraglottis: laryngeal edema
- Croup: laryngeal edema
- Abscess (intraoral, retropharygeal): distortion of the airway and
trismus
- Ludwig's angina: distortion of the airway and trismus
- Arthritis
- Rheumatoid arthritis: temporomandibular joint ankylosis,
cricoarytenoid arthritis, deviation of laynrx, restricted mobility
of cervical spine
- Ankylosing spondylitis: ankylosis of cervical spine, less
commonly ankylosis of temporomandibular joints, lack of
mobility of cervical spine
- Benign tumors
- Cystic hygroma, lipoma, adenoma, goiter: stenosis or distortion
of the airway, fixation of larynx or adjacent tissues secondary to
infiltration or fibrosis from irradiation
- Obesiture(s) of the maxillae, mandible and cervical vertebrae
sleep apnea
- Acromegaly: macroglossia, prognathism
- burns: edema of airway
track the cervical spine injury, laryngeal or
-

include the clinician-related risks; an acknowledgment that superior skills are called for in managing this extremely vulnerable patient population. Children with chronic medical problems require appropriate management and prediction of possible difficulties in management, which the preoperative assessment needs to focus on. Especially for infants, enquiries about prematurity, developmental milestones, and medical background check probing for possible congenital diseases of the cardiac and respiratory systems need to be made. Congenital cardiovascular malformations are frequently associated with many facial malformations, which require a thorough preoperative assessment. Another challenge that pediatric patients present, especially for the anesthesiologist, is the pediatric airway management due to a difference in the airway anatomy vis-a-vis adults. The relatively larger soft tissues in the oropharynx, a more cephalad and anterior position of the vocal cords and the larynx, and a shorter length of the trachea are among the few differences between an adult and pediatric airway anatomy [26]. These unique variations can result in airway management issues, including difficulties in sustaining a patent airway, mask ventilation, insertion of supraglottic airway devices, and endotracheal intubation.

\subsection{Assessment of the Elderly Patient}

Another vulnerable population is the geriatric age group. This is because older age is associated not only with dwindling functions of almost all organ systems but also an increase in the incidence of comorbidities. Further, as life expectancy increases, clinicians are likely to encounter a substantial number of elderly people in their practice, and it is conceivable that a large proportion of these may present for surgery. It is therefore vital that clinicians dealing with patients of this age group should be able to comprehend the alterations in physiology and pathophysiology that occur with aging. A diminished cardiopulmonary reserve puts this patient population under a higher risk of perioperative adverse events. Incidence of coronary artery disease is also prevalent in the geriatric population. Further, there is an increased risk of cognitive dysfunction such as dementia, delirium, and depression, which may be exacerbated in the acute postoperative phase. Elderly patients have impaired hepatic and renal metabolism and delayed clearance of drugs in the systemic circulation, which can cause increased sensitivity to intravenous anesthetics. Also important in relation to the elderly patient are several anatomical alterations, which can affect management. Predominant among these are changes in airway anatomy, such as nasopharyngeal fragility, macroglossia, microstomia, etc. [27]. These can result in difficulty in mask ventilation and placement of supraglottic airway devices and endotracheal tube.

\subsection{Specialist Consultation and Work-up}

The initial evaluation of the patient then leads to subsequent work-up, including obtaining consultations from other specialties if deemed necessary, re-evaluating the need for any additional investigations, taking steps to limit perioperative risks as deemed necessary, evolving an anesthetic plan, and getting informed consent from the patient. As more and more areas of concern may develop, further testing, specialist consultation, and varied approaches to risk management strategies may become imperative. As this dynamic process of comprehensive patient assessment evolves, revision of the anesthetic plan and eliciting additional specific terms of consent from the patient may also become inevitable.

Specialist consultations are needed when the particular condition being evaluated does not come under the purview of the training of the anesthesiologist or surgeon. Such consultations must be specific and try to elicit whether the 
patient is in the best possible condition with regard to his particular comorbidity to undergo the proposed intervention, or whether the malady for which consultation is being sought, can be further optimized and at what stage of the particular ailment the patient is at. When consultations are merely sent without stating specific objectives of such request, they might meet with a similarly nonspecific response from the consultant that the referral is being sent to, and hence proving to be of not much help for the clarification sought for. Thus, referrals to other disciplines need to be very specific, as to the expectations that the referring unit has of the evaluation and the advice that they should receive. The consultant to whom the referral goes now becomes an important component of the perioperative team. It is essential that this consultant must also appreciate his role in the assessment of the patient, and this would be possible by building support networks among disciplines. This will also save time in the perioperative period, since a few investigations that the consultant being referred to would advise, may be carried out prior to the referral itself. An example would be getting an ECG and echocardiogram done prior to referring a patient with significant coronary artery disease to the cardiologist. Thus, the requirement for circulation of information, among disciplines, is vital and is especially true with regard to communication between consultants. Often, communicating personally with the consultant to whom the patient is being referred to, can expedite the evaluation to a very great extent.

\subsection{The Process of Risk Assessment}

Following a thorough evaluation and perusal of the results of the investigations, the patient-specific risks are evident, while the surgery-specific risks need to be considered upon, the summation of both of which contribute to the overall risk to the patient. Technical factors such as fluid shifts, total blood loss, as well as the site of the surgical intervention, are components of the surgery-specific factors that contribute to the total risk assessment [28]. Oral and maxillofacial surgery being classified as head and neck surgery, would normally be considered as an intermediate risk surgery, unless undertaken as emergency or anticipated to have a risk of excessive blood loss, wherein it would be classified as a high-risk procedure. The correlation between expected blood loss and specific maxillofacial surgeries has been demonstrated [29]. With improving the safety of anesthesia equipment and medications, the historical dangers of anesthesia administration are quite low, and patient- and surgery-specific risk factors almost completely determine the overall risk to the patient $[30,31]$.

Once the overall risk of the patient is known and calculated, the next step would be to devise a strategy for risk management.

\subsection{Risk Reduction Strategies}

Risk reduction blueprints must be assessed and a risk-benefit ratio evaluated in performing any elective oral or maxillofacial surgical procedure. Alternative procedures and occasionally even avoiding any intervention could be considered when the overall risks in terms of morbidity and mortality to the patient are substantial. Obviously, the most critical step in risk reduction would be to get the patient in the best physical condition to undergo an interventional procedure. To this effect, modifiable risk factors should be addressed (e.g., anemia, hyperglycemia, hypertension) always ensuring that the risk-benefit ratio stays in the patient's favor. As an example, in a smoker who is to undergo an intermediate- or high-risk surgery, the obvious risk reduction strategy would be to quit smoking [32, 33].

This then allows the patient's body to reap the benefits of a simple risk reduction maneuver with the risk now decreasing to a degree that might cause a favorable impact on the morbidity and mortality otherwise expected. Appropriate medical management of the comorbidities might be successful in optimizing patients to a state where they can withstand the rigors of the surgical procedure. The particular anesthetic technique apart, there are at least five other areas that may be targeted as part of overall risk reduction strategies, namely: (a) premedication, (b) antiaspiration prophylaxis, (c) perioperative beta-blockade, (d) postoperative nausea and vomiting (PONV) prophylaxis and (e) effective postoperative pain management.

\subsubsection{Premedication [4]}

An increase in heart rate is the major factor increasing myocardial oxygen demand in the perioperative period. This makes appropriate anxiolysis and management of postoperative pain absolute vital cogs in the overall care of the patient undergoing surgery since anxiety and/or pain can set off a noxious cycle of events starting with tachycardia and hypertension, which could, in turn, result in perioperative myocardial infarction, the incidence of which is highest in the first $72 \mathrm{~h}$. Benzodiazepines induce anterograde amnesia, anxiolysis with mild sedation. Opiates were previously believed to be a vital part of preemptive analgesia, but later they were found to sensitize patients to pain. Clonidine may also be considered preemptive analgesia, especially when administered epidurally. However, the entire concept of pre-emptive analgesia, or antinociceptive treatment prior to initiation of the pain stimulus that prevents the establishment of altered processing of afferent input, that amplifies postoperative pain, is currently mired in controversy. Clonidine has a cardioprotective effect and also decreases the minimum alveolar concentration (MAC). Antihistamines may be used for their 
sedative and antiemetic properties. However, the clinician needs to know that promethazine, the most commonly prescribed antihistaminic, now carries an FDA warning for apnea and death in children. Anticholinergics are no longer used routinely, but rather to produce an antisialagogue effect when desirable (endoscopic procedures), sedation/amnesia, or to prevent reflex bradycardia.

\subsubsection{Fasting Guidelines}

Patients with a full stomach at the time of induction of anesthesia are perceived to be at high risk of aspiration of gastric contents into their respiratory tract, and also present difficulty with intubation if gastric contents are regurgitated at the time of airway manipulation. This may lead to acute lung injury manifested as pneumonitis, aspiration pneumonia, respiratory failure, or acute respiratory distress syndrome [34]. Following administration of a general anesthetic, patients can no longer have the ability to maintain a patent airway, respiration, or protective reflexes like gagging or coughing. The driving force for inducing aspiration is the barrier pressure, which is the difference between the gastroesophageal sphincter tone that opposes aspiration and the hydrostatic pressure within the stomach. In the presence of a negative barrier pressure, the possibility of regurgitation, vomiting, and aspiration is increased. One of the ways to maintain the barrier pressure positive during the vulnerable period of anesthesia is to instruct the patient to be "nil per oral" prior to surgery, such that the gastric hydrostatic pressure is kept low. What constitutes an appropriate period of fasting before anesthesia has been the subject of substantial research over several years, and current guidelines are based both on the nature of oral intake and the age of the patient $[35,36,37]$ (Table 2.5). These recommendations, however, need to be viewed as guidelines, rather than a set of inviolable instructions. The reason why these rules should not be looked upon as impregnable is because these were intended for normal patients. Individual situations may sometimes prevent these nil-per-oral guidelines from providing foolproof safety from aspiration. Conditions that inhibit gastric emptying, including diabetes, morbid obesity (body mass

Table 2.5 Preoperative fasting recommendations for healthy patients undergoing elective surgery

\begin{tabular}{l|l|}
\hline $\begin{array}{l}\text { Liquid and food intake } \\
\begin{array}{l}\text { Clear liquids (Water, tea, black coffee, fruit juice } \\
\text { without pulp, carbonated beverages) }\end{array}\end{array}$ & $\begin{array}{l}\text { Minimum fasting } \\
\text { period }\end{array}$ \\
\hline $\begin{array}{l}\text { Breast milk } \\
\text { Nonhuman milk, including infant milk formula }\end{array}$ & $4 \mathrm{~h}$ \\
\hline $\begin{array}{l}\text { Light meal (e.g., toast and clear liquids) } \\
\text { Regular or heavy meal (may include fried or fatty } \\
\text { food, meat) }\end{array}$ & $6 \mathrm{~h} \mathrm{~h}$ \\
\hline
\end{tabular}

index $[\mathrm{BMI}]$ >35), pregnancy, bowel obstruction, previous upper gastrointestinal surgery, and gastrointestinal disease such as gastric ulcer, scleroderma, etc. will all escalate the risk of aspiration and deserve as much attention that a full stomach does [38-40]. Another group of patients who are believed to be at high risk for aspiration comprises those with difficult airways, as these patients will require more prolonged airway manipulation and thus a greater time to intubation [41]. The airway remains unprotected and unsecured for a longer time and hence these patients may benefit from prophylaxis against aspiration. The preanesthetic evaluation needs to identify this special category of patients who appear to be at higher risk of aspiration, despite adhering faithfully to standard nil per oral guidelines and premedicate them for elective surgery. A prokinetic drug and an alkalinizing agent could reduce the complications from gastric aspiration by combating the corrosive acidic nature of gastric juice, even if aspiration does occur [3].

The most widely prescribed prokinetic in this regard is the dopamine-antagonist metoclopramide (10 mg orally/IV). However, this drug has the potential to cause extrapyramidal symptoms and should be used cautiously. The other pharmacological agent that increases the $\mathrm{pH}$ of gastric juice and makes it less acidic is a Histamine 2 (H2) receptor blocker, viz., ranitidine ( $150 \mathrm{mg}$ orally; $50 \mathrm{mg}$ IV) or famotidine (20 mg IV). Cimetidine is no longer used due to its ability to induce hepatic enzymes and influence the metabolism of several anesthetic agents. Usually, the prokinetic along with the $\mathrm{H} 2$ blocker is administered both on the night before and on the morning of the surgery. Administration of two doses each of both the medications guarantees adequate plasma levels required for an effective action. Lastly, the use of particulate antacids such as citric acid/sodium citrate $(30 \mathrm{ml})$ typically within $2 \mathrm{~h}$ prior to the anticipated induction of anesthesia also can neutralize the gastric acid, and reduce the incidence of chemical pneumonitis even if the aspiration occurs. However, its bitter taste and propensity to induce emesis necessitates that it be administered a substantial period prior to intubation.

\subsubsection{Perioperative Beta-Blocker Therapy}

Organ ischemia is a result of an imbalance between the oxygen supply and demand. The heart is especially vulnerable since myocardial work and oxygen demand are very tightly coupled. However, the precise level of myocardial demand at which it outstrips the supply is unclear. Hence, a patient who is susceptible to the development of myocardial ischemia needs to be managed perioperatively in a manner that keeps the demand lowest, while at the same time maximizing supply. Pharmacological manipulation of the myocardial demand is probably effected easier than a significant manipulation of 
the supply. Physiologically, tachycardia is the most important parameter that increases myocardial demand, with increases in systemic vascular resistance being the second most important [42-44]. Patients having a significant cardiovascular risk need a cardiologist's opinion for an evaluation of the extent of risk and preoperative optimization [6] (Table 2.6). Obviously, modifiable risk factors and reversible disorders need to be addressed and managed. The ACC recommends perioperative beta-blocker therapy for patients with two or more intermedi-

Table 2.6 Clinical predictors of increased perioperative cardiovascular risk

\section{Major clinical predictors (markers of unstable coronary artery} disease)

- Myocardial infarction <6 weeks: Delay elective surgery, consultation with cardiologist

- Unstable or severe angina (Canadian Cardiovascular Society class III or IV)

- Decompensated heart failure

- Significant arrhythmias (e.g., causing hemodynamic instability)

- Severe valvular heart disease (e.g., aortic or mitral valve stenosis with valve area $<1 \mathrm{~cm}^{2}$ )

- $\mathrm{CABG}$ or PTCA $<6$ weeks

Intermediate clinical predictors (markers of stable coronary artery disease)

- Previous Myocardial infarction $>6$ weeks but $<3$ months (> 3 months if complicated) based on the history or presence of pathologic Q waves on the Electrocardiogram

- Mild angina (Canadian Cardiovascular Society class I to II)

- Silent ischemia (Holter monitoring)

- Compensated heart failure, ejection fraction $<0.35$

- Post CABG or PTCA $>6$ weeks and $<3$ months, or $>6$ years, or on antianginal therapy

- Diabetes mellitus

- Renal insufficiency

Mild clinical predictors (increased probability of coronary artery

disease)

- Familial history of coronary artery disease

- Age $>70$ years

- ECG abnormalities (arrhythmias, LVH, Left bundle branch block)

- Low functional capacity

- History of stroke

- Uncontrolled systemic hypertension

- Hypercholesterolemia

- Smoking

- Post infarction (> 3 months, asymptomatic without treatment

- Post CABG or PTCA ( $>3$ months and $<6$ years, and no symptoms of angina nor on antianginal therapy)

Major clinical predictors: All elective operations should be postponed and the patients properly investigated and treated. Intermediate clinical predictors: Proof of well-established but controlled coronary artery disease. Further risk stratification and optimization in consultation with cardiologist.

Minor clinical predictors: Increased probability of coronary artery disease, but not of an increased perioperative risk. Further risk stratification and optimization in consultation with cardiologist. Emergency procedures: Proceed for surgery with perioperative surveillance and postoperative risk stratification and risk factor management.

$C A B G$ Coronary Artery Bypass Grafting, PTCA Percutaneous Transluminal Coronary Angioplasty, LVH Left Ventricular Hypertrophy ate risk factors or a single major risk factor, especially if being posted for an intermediate-to-high-risk surgical procedure [6]. The evaluating physician can be requested to assess the benefits of beta-blockade in the patient scheduled for maxillofacial surgery. The commonest way of introducing betablockade is with the drug metoprolol for a target basal heart rate of 60-65 beats per minute (bpm), and to sustain the rates at no more than $85 \mathrm{bpm}$ during the perioperative period. Betablockade should, however, be started well before the surgery so that ample time is available to adjust the dose to the target heart rate and address any side effects that may develop. The administration of a beta-blocker just prior to an emergency surgery is controversial as this has been observed to be associated with a greater risk of perioperative strokes [45, 46]. More research is needed to clearly identify which patient subsets might benefit most from the beta-blockade, and in whom the side effects may be substantial.

\subsubsection{PONV Prophylaxis [47]}

Oral and Maxillofacial surgeries are generally not considered as high risk for the incidence of postoperative nausea and vomiting (PONV) [48]. Risk factors, including female gender, previous history of PONV or motion sickness, nonsmoking status, and younger age patients, have been associated with an increased incidence and severity of PONV [49]. Therefore, the preoperative assessment should be sensitive to the presence of these indicators and must devise a plan for offering pharmacological prophylaxis to this patient population. The plan for anesthesia devised preoperatively should also take into consideration that use of volatile anesthetics, opioids, and nitrous oxide may be associated with higher PONV incidence, and thus influence the choice of anesthetic agents used. The choices for PONV prophylaxis are between 5HT3 antagonists (ondansetron, granisetron, palonosetron, etc.), dexamethasone, subanesthetic doses of propofol, scopolamine patches, etc. [50]. Generally, a combination of agents seems to work much better than a single agent.

\subsubsection{Plan for Postoperative Analgesia [51]}

Regional anesthesia techniques, including peripheral nerve blocks, are excellent in terms of providing quality analgesia postoperatively, but they do have their own set of risks and may cause discomfort from the ensuing numbness. Hence, it is important that the patient is taken into confidence about the risks and benefits of this modality of pain relief, while also offering alternative approaches that could be opted for. Local anesthesia in adequate concentrations and volume is essential for a pain-free postoperative period. Contrary to earlier practices, the mixing of local anesthetic solutions to 
apparently harness the best pharmacological effects of both is an approach, which is currently being discouraged. In fact, a successful preoperative nerve block may also be used so as to minimize the incidence of tachycardia during the intraoperative noxious stimuli, and then repeated at the culmination of the procedure, so that the effect can extend into the postprocedure period. This option can be employed by the maxillofacial surgeon resultant to their expertise in regional nerve blocks of the orofacial region. Opioids have traditionally been the workhorse for postoperative analgesia. Intravenous patient-controlled analgesia is an attractive option for the postoperative pain control for the inpatient in whom the effects of the nerve blocks are expected to wane after a few hours. However, there is accumulating evidence that a multimodal approach to pain management works best, with the intent of reducing the side effects of opioids and improving pain scores. Enhanced recovery after surgery (ERAS) protocols to minimize postsurgery hospital stay are gaining wider acceptance, and an important part of such protocols is the inclusion of multimodal opioid-sparing pain management protocols [52].

\subsection{Preoperative Decision Making and Obtaining Informed Consent: The Team Concept [53]}

The preoperative plan blends the patient's requirements, the competence of the perioperative team, and the infrastructure provided by the hospital to get the particular intervention done. This, it is expected, would help achieve the favorable outcome of the intervention. Quite often, the optimization of all of these conditions may not always be possible. The onus then is upon both the patient and the operating team to take a decision on what may be the acceptable course of action. Whether to pursue the performance of a particular intervention or not is, therefore, also an integral part of the preoperative decision-making process.

The final element of a comprehensive preoperative assessment is the process of obtaining a valid, written, and informed consent. Obviously, this process can be initiated, only after a thorough risk estimation has been made, based on the preceding evaluation of the patient, and a tentative anesthetic and surgical plan have been evolved, including strategies for management of postoperative pain. For all practical purposes, an informed consent necessitates presentation of the final residual risk that the patient has, which cannot be further optimized, and its expected interaction with the proposed surgical intervention. The main players in the process of obtaining informed are obviously the surgeon, anesthesiologist, and the patient. Typically, any of them has the autonomy to reject or to suggest modifications to the proposed sequence for the intervention. It is important to note that informed consent is a process of ensuring the patient understands the risks and benefits of the available surgical and anesthetic options. It is not merely signing a consent form. The capacity of a patient refers to the ability to make decisions about their care, and to decide whether to agree to, or refuse, an examination, investigation, or treatment. To have the capacity, a patient must be able to understand and retain information regarding the treatment, evaluate the risks and benefits of treatment, reach a decision regarding their course of treatment, and communicate their decision to the clinician [54]. A patient undergoing surgery is understandably apprehensive, and hence the process of imparting information to him while taking consent must be handled professionally, yet with the utmost sensitivity [55]. It is important that the anesthesiologist and the surgeon should have thrashed out issues relating to perioperative care and broken common ground on all aspects before involving the patient (Table 2.7).

Following discussion and concordance between the surgeon and the anesthesiologist on the course of action, the patient is taken into confidence, informed about the procedure, and his/her consent for the same, sought. The information provided to the patient includes the nature of the risk involved, the benefits, and the alternatives to the proposed plan. If the proposal or any particular element of it is unacceptable to the patient, the operating team must be ready for course correction and should be able to come up with alternatives, which would be acceptable to the patient. However, if no substitute plan can be offered, and the patient is resolute in not accepting the proposed one, there is no option than to abort the proposed surgery. Options, such as referrals to other surgical teams, with different expertise or set-ups that may address the patient's concerns better and satisfy his expectations can be considered. Consequently, acquiring informed consent is a highly interactive process, with the patient being the pivot, to whom the risk-benefit ratio is projected taking into consideration his/her wishes and needs. The Association of Oral and Maxillofacial Surgeons of India consent forms can serve as a useful template for consent taking in one's clinical practice [56].

Table 2.7 Points of concurrence between surgeon and anesthesiologist before seeking informed consent from the patient

\footnotetext{
- Diagnosis

- Nature and purpose of the proposed treatment or procedure

- Risks, complications, and consequences of the proposed treatment or procedure

- Alternatives to proposed treatment or procedure

- The consequences if the proposed treatment or procedure is refused

- Benefits and expected results if the proposed treatment or procedure is accepted

- Conflicts of interest

- Who will be providing the treatment or procedure
} 


\subsection{Conclusion}

"Do not operate on a stranger" is the most important mantra to be followed by any surgeon. The preoperative evaluation of oral and maxillofacial surgery patient comprises a critical part of the perioperative period and hence requires a watchful and staged outlook to risk identification and management, putting in place a plan for anesthesia, surgery, and postsurgical pain management. There is no substitute for a properly performed and detailed history and physical examination. Literature suggests that most clinically relevant conditions are recognized during the history and physical examination without the need for further laboratory testing. The surgical team needs to ascertain the risks involved with the comorbidities that the patient may be suffering from, and be able to juxtapose this against the procedure-specific risks to identify the overall risk of the procedure in that particular patient. Risk management and optimization strategies involve the rational use of adjunctive laboratory and imaging studies and professional opinions of consultants of other specialties. This helps evaluate the degree of physiological decompensation a patient might be having due to his comorbidities, and whether this could be either reversed or ameliorated. Acquiring informed consent from the patient involves the development of consensus between the surgeon and anesthesiologist first, with regard to the perioperative plan, irrespective of whether the consent forms for anesthesia and surgery are the same or different documents. This proposed plan, which has the concurrence of both the surgeon and the anesthesiologist, is then presented to the patient along with clear information about the risks and benefits of different clinical strategies, such that the patient can make his up his/her mind, or else request refinement of the proposed plan. This thorough process of preoperative evaluation, it is expected, would be able to keep morbidity and mortality related to the maxillofacial surgery, to an absolute minimum.

\section{References}

1. Galway UA. Preoperative evaluation. In: Sikka PK, Beaman ST, Street JA, editors. Basic clinical anesthesia. New York: Springer; 2015. p. 7-15.

2. Hilditch WG, Asbury AJ, Jack E, McGrane S. Validation of a preanaesthetic screening questionnaire. Anaesthesia. 2003;58:874.

3. Wijeysundera DN, Sweitzer BJ. Preoperative evaluation. In: Miller RD, editor. Miller's anesthesia. 8th ed. Philadelphia: Elsevier Saunders; 2015. p. 1085-155.

4. Hata TM, Hata JS. Preoperative patient assessment and management. In: Barash PG, Cullen BF, Stoelting RK, Cahalan MK, Stock MC, Ortega R, Sharar SR, Holt NF, editors. Clinical anesthesia. 8th ed. Philadelphia: Wolters Kluwer; 2017. p. 1479-544.

5. Gupta A, Shiveley T. The preoperative patient evaluation. In: Ehrenfeld JM, Urman RD, Segal S, editors. Anesthesia student survival guide-a case based approach. 2nd ed. New York: Springer; 2016. p. 89-106.
6. Fleisher LA, Beckman JA, Brown KA, Calkins H, Chaikpf E, Fleischmann K, et al. ACC/AHA 2007 Guidelines on Perioperative Cardiovascular Evaluation and Care for Noncardiac Surgery: a Report of the American College of Cardiology/American Heart Association Task Force on Practice Guidelines (Writing Committee to Revise the 2002 Guidelines on Perioperative Cardiovascular Evaluation for Noncardiac Surgery). Circulation. 2007;116:e418-99.

7. Owens WD. American Society of Anesthesiologist physical classification system is not a risk classification system. Anesthesiology. 2001;94:378.

8. Henry A, Dewi F, Atan R, Patel N, Bhatia S. Pre-operative investigations in patients undergoing oral and maxillofacial procedures. Oral Surg. 2016;9:155-9.

9. Tasse JL, Janzen ML, Ahmed NA, Chung RS. Screening laboratory and radiology panels for trauma patients have low utility and arenot cost effective. J Trauma. 2008;65:1114-6.

10. Bryson GL, Wyand A, Bragg PR. Preoperative testing is inconsistent with published guidelines and rarely changes management. Can J Anaesth. 2006;53:236-41.

11. Smetana GW, Macpherson DS. The case against routine preoperative laboratory testing. Med Clin North Am. 2003;87:7-40.

12. Weinberger SE. Providing high-value, cost-conscious care: a critical seventh general competency for physicians. Ann Intern Med. 2011;155:386-8.

13. Benarroch-Gampel J, Riall TS. What laboratory tests are required for ambulatory surgery? Adv Surg. 2013;47:81-98.

14. Feely MA, Collins CS, Daniels PR, Kebede EB, Jatoi A, Mauck KF. Preoperative testing before noncardiac surgery: guidelines and recommendations. Am Fam Physician. 2013;87(6):414-8.

15. Czoski-Murray C, Lloyd Jones M, McCabe C, Claxton K, Oluboyede Y, Roberts J, et al. What is the value of routinely testing full blood count, electrolytes and urea, and pulmonary function tests before elective surgery in patients with no apparent clinical indication and in subgroups of patients with common comorbidities: a systematic review of the clinical and cost-effective literature? Health Technol Assess. 2012;16(i-xvi):1-159.

16. Parameters of Care; AAOMS clinical practice guidelines for oral and maxillofacial surgery (AAOMS ParCare), 6th ed; 2017; J Oral Maxillofac Surg 75:Suppl 1e12-1e33.

17. Fattahi T. Perioperative laboratory and diagnostic testing-what is needed and when? Oral Maxillofac Surg Clins North Am. 2006;18:1-6.

18. National Institute for Health and Care Excellence (NICE) guidelines. Routine pre-operative tests for elective surgery 2016. www. nice.org.uk/guidance/ng45.

19. Apfelbaum JL, Hagberg CA, Caplan RA, Blitt CD, Connis RT, Nickinovich DG, et al. Practice guidelines for management of the difficult airway: an updated report by the American Society of Anesthesiologists Task Force on Management of the Difficult Airway. Anesthesiology. 2013;118:251-70.

20. Prakash S, Mullick P, Bhandari S, Kumar A, Gogia AR, Singh R. Sternomental distance and sternomental displacement as predictors of difficult laryngoscopy and intubation in adult patients. Saudi J Anaesth. 2017;11:273-8.

21. O'Dell K. Predictors of difficult intubation and the otolaryngology perioperative consult. Anesthesiol Clin. 2015;33:279-90.

22. Reed MJ, Dunn MJG, McKeown DW. Can an airway assessment score predict difficulty at intubation in the emergency department? Emerg Med. 2005;22:99-102.

23. Murphy Michael F, Walls RM. Identification of the difficult and failed airway. In: Walls RM, Michael F, editors. Emergency airway management. 3rd ed. Philadelphia: Lippincott Williams \& Wilkins; 2008. p. 82-93.

24. Advanced Trauma Life Support (ATLS) Student course manual. Airway and ventilatory management. 9th ed. Chicago IL, 2012. American College of Surgeons Committee on Trauma: 36. 
25. Mallampati SR, Gatt SP, Gugino LD, Desai SP, Waraksa B, Freiberger D, et al. A clinical sign to predict difficult tracheal intubation: a prospective study. Can Anaesth Soc J. 1985;32:429-34.

26. Advanced Trauma Life Support (ATLS) student course manual. Pediatric trauma. 9th ed. Chicago IL, 2012. American College of Surgeons Committee on Trauma: 251.

27. Advanced Trauma Life Support (ATLS) student course manual. Geriatric trauma. 9th ed. Chicago IL, 2012. American College of Surgeons Committee on Trauma: 275.

28. Glance LG, Lustik SJ, Hannan EL, Osler TM, Mukamel DB, Qian F, et al. The Surgical Mortality Probability Model: derivation and validation of a simple risk prediction rule for noncardiac surgery. Ann Surg. 2012;255:696-702.

29. Akinbami BO, Onajin-Obembe B. Assessment of intraoperative blood loss during oral and maxillofacial surgical procedures in a Nigerian tertiary health care center. J Blood Transfus. 2014;2014:301467.

30. Lanier WL. A three-decade perspective on anesthesia safety. Am Surg. 2006;72:985-9.

31. Botney R. Improving patient safety in anesthesia: a success story? Int J Radiat Oncol Biol Phys. 2008;71:S182-6.

32. Rodrigo C. The effects of cigarette smoking on anesthesia. Anesth Prog. 2000;47:143-50.

33. Warner DO. Perioperative abstinence from cigarettes: physiologic and clinical consequences. Anesthesiology. 2006;104:356-67.

34. Warner MA, Warner ME, Weber JG. Clinical significance of pulmonary aspiration during the perioperative period. Anesthesiology. 1993;78:56-62.

35. Apfelbaum JL, Caplan RA, Connis RT, Epstein BS, Nickinovich DG, Warner MA. American Society of Anesthesiologists Committee. Practice guidelines for preoperative fasting and the use of pharmacologic agents to reduce the risk of pulmonary aspiration: application to healthy patients undergoing elective procedures: an updated report by the American Society of Anesthesiologists Committee on Standards and Practice Parameters. Anesthesiology. 2011;114:495-511

36. Brady M, Kinn S, Stuart P. Preoperative fasting for adults to prevent perioperative complications. Cochrane Database Syst Rev. 2003;4:CD004423.

37. Stuart PC. The evidence base behind modern fasting guidelines. Best Pract Res Clin Anaesthesiol. 2006;20:457-69.

38. Fleisher LA, Mythen M. Anesthetic implications of concurrent diseases. In: Miller RD, editor. Miller's anesthesia. 8th ed. Philadelphia: Elsevier Saunders; 2015. p. 1156-225.

39. Krishnan B. Obese oral and maxillofacial surgical patient. J Craniofac Surg. 2009;20:53-7.
40. Hoefnagel A, Yu A, Kaminski A. Anesthetic complications in pregnancy. Crit Care Clin. 2016;32:1-28.

41. Neuman MD, Fleisher LA. Risk of anesthesia. In: Miller RD, editor. Miller's anesthesia. 8th ed. Philadelphia: Elsevier Saunders; 2015. p. 1056-84.

42. Fox KM, Ferrari R. Heart rate: a forgotten link in coronary artery disease? Nat Rev Cardiol. 2011;8:369-79.

43. Tardif JC. Heart rate as a treatable cardiovascular risk factor. $\mathrm{Br}$ Med Bull. 2009;90:71-84.

44. Braunwald E. Control of myocardial oxygen consumption: physiologic and clinical considerations. Am J Cardiol. 1971;27:416-32.

45. Lindholm LH, Carlberg B, Samuelsson O. Should beta blockers remain first choice in the treatment of primary hypertension? A meta-analysis. Lancet. 2005;366:1545-53.

46. Bangalore S, Messerli FH, Kostis JB, Pepine CJ. Cardiovascular protection using betablockers: a critical review of the evidence. J Am Coll Cardiol. 2007;50:563-72.

47. Fowler MA, Spiess BD. Postanesthesia recovery. In: Barash PG, Cullen BF, Stoelting RK, Cahalan MK, Stock MC, Ortega R, Sharar SR, Holt NF, editors. Clinical anesthesia. 8th ed. Philadelphia: Wolters Kluwer; 2017. p. 3854-918.

48. Alexander M, Krishnan B, Yuvraj V. Prophylactic antiemetics in oral and maxillofacial surgery: a requiem? J Oral Maxillofac Surg. 2009;67:1873-7.

49. Habib AS, Gan TJ. Evidence-based management of postoperative nausea and vomiting: a review. Can J Anaesth. 2004;51:326-41.

50. Cao X, White PF, Ma H. An update on the management of postoperative nausea and vomiting. J Anesth. 2017;31:617-26.

51. White PF, Kehlet H, Neal JM, Schricker T, Carr DB, Carli F, et al. The role of the anesthesiologist in fast-track surgery: from multimodal analgesia to perioperative medical care. Anesth Analg. 2007;104:1380-96.

52. Misiołek H, Cettler M, Woroń J, Wordliczek J, Dobrogowski J, Mayzner-Zawadzka E. The 2014 guidelines for post-operative pain management. Anaesthesiol Intensive Ther. 2014;46:221-44.

53. Barry MJ, Edgman-Levitan S. Shared decision making-pinnacle of patient-centered care. N Engl J Med. 2012;366:780-1.

54. General Medical Council (GMC). Consent: patients and doctors making decisions together [online]. GMC, 2008. Available: http:// www.gmc-uk.org/static/documents/content/Consent-English0414. pdf

55. Sjöling M, Nordahl G, Olofsson N, Asplund K. The impact of preoperative information on state anxiety, postoperative pain and satisfaction with pain management. Patient Educ Couns. 2003;51:169-76.

56. Association of Oral and Maxillofacial Surgeons of India (AOMSI): https://www.aomsi.com/WebPages/downloads.aspx

Open Access This chapter is licensed under the terms of the Creative Commons Attribution 4.0 International License (http://creativecommons. org/licenses/by/4.0/), which permits use, sharing, adaptation, distribution and reproduction in any medium or format, as long as you give appropriate credit to the original author(s) and the source, provide a link to the Creative Commons license and indicate if changes were made.

The images or other third party material in this chapter are included in the chapter's Creative Commons license, unless indicated otherwise in a credit line to the material. If material is not included in the chapter's Creative Commons license and your intended use is not permitted by statutory regulation or exceeds the permitted use, you will need to obtain permission directly from the copyright holder.

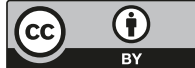

